Illinois State University

ISU ReD: Research and eData

Theses and Dissertations

6-6-2016

\title{
The Effects of Two Different Resistance Training Protocols with Similar Volume on Non-Uniform Muscle Hypertrophy
}

Vince Bendotti

Illinois State University, vlbendo@ilstu.edu

Follow this and additional works at: https://ir.library.illinoisstate.edu/etd

Part of the Kinesiology Commons

\section{Recommended Citation}

Bendotti, Vince, "The Effects of Two Different Resistance Training Protocols with Similar Volume on NonUniform Muscle Hypertrophy" (2016). Theses and Dissertations. 561.

https://ir.library.illinoisstate.edu/etd/561

This Thesis is brought to you for free and open access by ISU ReD: Research and eData. It has been accepted for inclusion in Theses and Dissertations by an authorized administrator of ISU ReD: Research and eData. For more information, please contact ISUReD@ilstu.edu. 


\title{
THE EFFECTS OF TWO DIFFERENT RESISTANCE TRAINING PROTOCOLS
}

\section{WITH SIMILAR VOLUME ON NON-UNIFORM MUSCLE HYPERTROPHY}

\author{
Vincent L. Bendotti
}

\section{Pages}

Resistance training programs utilize variables such as exercise volume, intensity, muscular activation, and tension in order to obtain specific hypertrophic adaptations. The challenge of designing a hypertrophy-centric program is understanding the non-uniform adaptations of skeletal muscle. PURPOSE: The purpose of this study was to observe the impact of two volume-equated, whole body exercise protocols over 9 weeks utilizing 9 different compound exercises on non-uniform muscle adaptations. METHODS: Fourteen $($ age $=20.9 \pm 1.3$ years; body mass $=84.0 \pm 11.1 \mathrm{~kg}$; fat free mass $=67.3 \pm 5.7$ $\mathrm{kg}$ ), well-trained men randomly assigned to a hypertrophy based protocol (HG) or a strength based protocol (SG). The HG performed 3 sets of 10 repetitions, with a rest period of 90 seconds between sets $(n=8)$. The SG performed 7 sets of 3 repetitions with 3 minutes of rest between sets $(n=6)$. Pre, mid, and post testing of proximal and distal muscle thickness measurements (MT) were taken on each subject's forearm flexors (FF), forearm extensors (FE), rectus femoris (RF), and vastus lateralis (VL). Each measurement was taken on the right side of the body to develop consistency across all of the subjects. RESULTS: After 9 weeks, statistically significant differences between SG 
and HG at both the Proximal Forearm Flexors (PFF) and Distal Forearm Extensors (DFE). There were significant differences in the trajectory of change between the groups $(p=.040)$ and throughout pre-, mid- and post-testing $(p=.034, .001, .000)$ of the PFF. Between the groups, DFE was statistically different at post-testing $(p=.036)$ and for overall trajectory of change $(p=.040)$. However, there were no statistically significant differences between groups in the other muscle thickness measurements.

CONCLUSIONS: When a strength and hypertrophy full-body resistance training programs are equated for volume, non-uniform hypertrophy occurs in the distal FE and proximal FF MT sites of the forearm, but fails to be seen in the quadriceps MT sites.

KEYWORDS: Strength, Stress, Thickness 
THE EFFECTS OF TWO DIFFERENT RESISTANCE TRAINING PROTOCOLS WITH SIMILAR VOLUME ON NON-UNIFORM MUSCLE HYPERTROPHY

VINCENT L. BENDOTTI

A Thesis Submitted in Partial Fulfillment of the Requirements for the Degree of

MASTER OF SCIENCE

School of Kinesiology and Recreation

ILLINOIS STATE UNIVERSITY

2016 
(C) 2016 Vincent L. Bendotti 
THE EFFECTS OF TWO DIFFERENT RESISTANCE TRAINING PROTOCOLS WITH SIMILAR VOLUME ON NON-UNIFORM MUSCLE HYPERTROPHY

VINCENT L. BENDOTTI

COMMITTEE MEMBERS:

Kelly R. Laurson, Chair

Daniel J. Dodd

Noelle M. Selkow 


\section{ACKNOWLEDGMENTS}

I would like to express my gratitude to Cory Worthey for welcoming me to work beside him during this experiment. I would not have been able successfully complete this project without his continuous hours of help, perseverance and hard work.

Next, I would like to whole-heartedly thank Dr. Kelly Laurson and his genuine interest in what I wanted to achieve with this project and my degree. His advice and countless hours of guidance were crucial throughout this process.

I would also like to extend my appreciation to Dr. Daniel Dodd for his expertise in resistance training, his utmost confidence in myself and for always extending a helping hand when I needed it most.

Next, I would like to thank Dr. Noelle Selkow for her expertise with the diagnostic ultrasound imaging and for everything she does as the KNR Graduate Program Director.

I am also incredibly appreciative of Dr. Robert Wildman at Dymatize Nutrition for providing the subjects with protein. Lastly, I am simply grateful to the participants and the research team for their hard work throughout the course of this study.

V. L. B. 


\section{CONTENTS}

ACKNOWLEDGMENTS $\quad$ i

CONTENTS

TABLES $\quad$ iv

\section{CHAPTER}

I. THE EFFECTS OF TWO DIFFERENT RESISTANCE TRAINING PROTOCOLS WITH SIMILAR VOLUME ON NON-UNIFORM MUSCLE HYPERTROPHY

Introduction $\quad 1$

Methods 3

Subjects 3

Resistance Training Procedures $\quad 4$

Muscle Thickness Measurements $\quad 6$

Muscular Strength Assessments $\quad 7$

Statistical Analyses $\quad 7$

$\begin{array}{lr}\text { Results } & 8\end{array}$

Muscle Thickness $\quad 8$

$\begin{array}{ll}\text { Muscular Strength } & 10\end{array}$

$\begin{array}{lr}\text { Discussion } & 10\end{array}$

$\begin{array}{ll}\text { Practical Application } & 15\end{array}$

II. EXTENDED LITERATURE REVIEW 16

Introduction $\quad 16$

Volume $\quad 18$

$\begin{array}{ll}\text { Intensity } & 20\end{array}$

Non-Uniform Hypertrophy 22 
Gaps in the Literature

REFERENCES 


\section{TABLES}

Table $\quad$ Page

1. Baseline Descriptive Statistics 3

2. Order of Exercises for Both Experimental Training Groups 4

3. Muscle Thickness Measurements and Percent Change 9 


\section{CHAPTER I}

\section{THE EFFECTS OF TWO DIFFERENT RESISTANCE TRAINING PROTOCOLS}

WITH SIMILAR VOLUME ON NON-UNIFORM MUSCLE HYPERTROPHY

\section{Introduction}

Populations of all types seek to achieve aesthetic, strength, balance, and other health benefits associated with resistance training (RT). One of the most sought after adaptations is skeletal muscle hypertrophy. Muscular hypertrophy occurs when skeletal muscle is subjected to an overload stimulus, it causes perturbations in myofibers and the related extracellular matrix (Schoenfeld, 2010). This sets off a chain of myogenic events that ultimately leads to an increase in the size and amounts of the myofibrillar contractile proteins actin and myosin, and the total number of sarcomeres in parallel. This, in turn, augments the diameter of individual fibers and thereby results in an increase in muscle cross-sectional area (Schoenfeld, 2010). In humans, resistance training is the most commonly accepted practice in producing an adequate stimulus to induce skeletal muscle hypertrophy (Antonio, 2000). Although past research has shown the hypertrophic response is maximized by using moderate (40-80\% of one-repetition max (1RM)) loads with relatively brief rest between sets and heavy loads (80-100\% 1RM) with lengthy rest intervals induce optimal strength gains; current research has illustrated significant hypertrophy can be achieved using heavy loads and lengthy rest periods (Schoenfeld, 2014). 
Understanding the variables impacting muscular adaptation is crucial in developing a successful resistance training protocol. Correct manipulation of volume, intensity, exercise selection, and rest periods result in muscular hypertrophy (Fisher, 2013); but current research illustrates that hypertrophy in skeletal muscle occurs in a complex and non-uniform fashion. Longitudinal resistance-training studies demonstrate that individual muscle as well as groups of synergist muscles adapt in a regional-specific manner (Antonio, 2000). These non-uniform adaptations fail to be fully understood, and variables associated with hypertrophy may need to be reexamined (Antonio, 2000).

Research has compared the effects of high-intensity strength and low-intensity hypertrophy training routines in untrained men, but studies including well-trained men are scarce (Schoenfeld, 2014). One study using well trained men found that the strengthoriented group experienced greater strength gains and hypertrophic gains in comparison to the hypertrophy-oriented group, though volume was not equated between the groups (Mangine, 2015). A study comparing equal volume between high and low intensity style protocols found that hypertrophy occurred similarly between the groups but the high intensity group had significantly higher strength gains (Schoenfeld, 2014). The purpose of this study was to further examine how two different volume-equated, total-body resistance training protocols impact non-uniform muscular adaptions in forearm and quadriceps muscle groups. While past research compared one muscle thickness (MT) measurement between strength and hypertrophy groups, this study looks at multiple MT sites on both the upper- and lower-body segments. 


\section{Methods}

\section{Subjects}

Fourteen college-aged men $($ age $=20.9 \pm 1.29$ years; body mass $=84.0 \pm 11.09$ $\mathrm{kg}$; fat free mass $=67.3 \pm 5.66 \mathrm{~kg}$ ) volunteered for this study. The subjects were free of any present musculoskeletal injuries and allergies to soy or whey protein. Subjects denied any use of anabolic steroids or other legal or illegal agents known to enhance muscular hypertrophy during the last year. All participants were well-trained and reported consistently participating in resistance training 3 times per week for a minimum of 1 year (RT experience $=4.5 \pm 2.87$ years).

All participants were pair-matched in relation to baseline strength and then randomly assigned to either the strength training group (SG) or the hypertrophy training group (HG). Approval was obtained from the Institutional Review Board. Informed consent was obtained from all participants before beginning the study. Baseline descriptive statistics are provided in Table 1.

Table 1

Baseline Descriptive Statistics

\begin{tabular}{lll}
\hline \multicolumn{1}{c}{ Descriptor } & \multicolumn{1}{c}{ Strength Group } & Hypertrophy Group \\
\hline Age (y) & $21.7 \pm 1.2$ & $20.3 \pm 1.0$ \\
Weight $(\mathrm{kg})$ & $83.4 \pm 9.6$ & $84.5 \pm 12.7$ \\
Fat-free mass (kg) & $69.2 \pm 4.5$ & $65.9 \pm 6.3$ \\
Resistance training & $5.8 \pm 3.7$ & $3.5 \pm 1.8$ \\
$\quad$ Experience (y) & & \\
\hline
\end{tabular}




\section{Resistance Training Procedures}

The subjects were consistently instructed to abstain from participating in any outside resistance-type training throughout their involvement during the study. The weight-training protocol entailed 3 exercises per session, 3 days per week, summing to 9 diverse exercises a week. The exercises included 3 upper-body pressing movements (flat barbell bench press, incline barbell bench press, and decline Smith machine bench press), 3 upper-body pulling movements (wide-grip cable pull-down, plate-loaded machine row, and close-grip cable pull-down), and 3 lower-body movements (barbell back squat; barbell glute bridge; and unilateral barbell lunges). All subjects executed the same exercises in the same order. This resulted in a total-body exercise routine for the subjects during each training session. Each of the three sessions can be found in Table 2.

Table 2

Order of Exercises for Both Experimental Training Groups

\begin{tabular}{lll}
\hline \multicolumn{1}{c}{ Session 1 } & \multicolumn{1}{c}{ Session 2 } & \multicolumn{1}{c}{ Session 3 } \\
\hline Flat Bench Press & Incline Bench Press & Decline Smith Press \\
Barbell Back Squat & Glute Bridge & Unilateral Barbell Lunges \\
Wide-grip Pull-down & Plate-loaded Row & Close-grip Pull-down \\
\hline
\end{tabular}


Participants completed 3 non-consecutive days of training a week, for a total of 9 weeks. Each of the sets were deemed complete when muscular failure was achieved, until the subjects were unable to perform a complete repetition executing proper form. Muscle failure was used to control for set termination criteria for all subjects. Similar total volume load (total reps x load) was achieved amongst both RT routines. The fifth week of training consisted of a deloading week that used $75 \%$ of the previous week's workloads for 2 sessions and the third session was replaced by a re-test of all strength and anthropometric measurements. Workloads were modified set-to-set and week-to-week. If either group achieved less than their designated repetitions, the resistance was lightened for the next set. In the case of achieving more than their goal repetitions, the resistance was increased for the next set. This encouraged maximum exertion while maintaining the appropriate repetition ranges. There was direct supervision of the subjects by the research team that consisted of two nationally-certified personal trainers at minimum during all training sessions to ensure proper execution of all exercises during the study.

The exercises were arranged so that the dominant muscle was not directly trained from set to set. The sessions consisted of an upper-body push, lower-body, and upperbody pull exercise arrangement. Subjects in HG performed moderate repetitions consisting of 8-12 repetitions with the target range of 10 repetitions. The subjects in SG completed low repetitions that consisted of 2-4 repetitions with the projection of 3 repetitions. The standard guidelines for rest included 3 minutes for the SG and 90 seconds for the HG (American College of Sports Medicine, 2009). 


\section{Muscle Thickness Measurements}

The subjects were instructed to lie in the anatomical position during each MT measurement. In the midline of the muscle or muscle group of interest, the MT measurement sites were marked at one-third and two-thirds of the length of the limb. These sites would allow for observations to be seen near the origin and insertion of the muscle, but not close enough that there would be a higher ratio of tendon to muscle. This also remained close enough to the mid-point to negate a MT site of one-half of the length of the muscle. The subjects were instructed to lie in the supine or prone positions and the measurements were taken on the right side of their body to ensure consistency between all subjects. A water-soluble transmission gel was placed on the subjects and the Terason T3000 M-series diagnostic ultrasound imaging system (Terason, Burlington, MA, USA) that utilizes a B-mode ultrasonography at $12 \mathrm{MHz}$ was used by a trained technician. Without disturbing the skin or subcutaneous adipose tissue, the probe was carefully placed parallel to the muscle or muscle group of interest. Images were utilized to measure from the subcutaneous adipose tissue-muscle interface to the muscle-bone interface for the forearm flexors and extensors. Measurements for the vastus lateralis and rectus femoris muscles were taken from the subcutaneous adipose tissue-muscle interface to the deep aponeurosis that divides the superficial rectus femoris and vastus lateralis muscles from the vastus intermedius. Due to the thickness of the lower-body muscles and the limiting diagnostic ultrasonography imaging unit depth of $7 \mathrm{~cm}$, this technique was chosen. 


\section{Muscular Strength Assessments}

The subjects performed the free weight exercises, barbell bench press (BP) and barbell back squat (BS), this assessed upper- and lower-body strength. To ensure proper 1RM testing procedures, the National Strength and Conditioning Association (NSCA) protocols were used (Baechle, 2008). Before both of the assessments, the subjects performed a warm-up that included 5-10 minutes of light cardiovascular exercise. They then proceeded to complete progressive loading warm-up sets of 5 repetitions at $50 \%$ of their $1 \mathrm{RM}$ and 2-3 repetitions at 60-80\% 1RM. Each 1RM attempt were executed with increasing weight after successful attempts, with 3-5 minutes of rest between each attempt. A successful 1RM BS was achieved when the subjects reached a position of parallel between femur and the floor and successfully returned to the starting position. A member of the research group with direct lateral view determined if the subjects reached parallel. To reach a successful 1RM BP, the subject must maintain five points of contact during the 1RM attempt (head, upper back, buttocks, and both feet). 1RM BS attempts were completed before the 1RM BP repetitions. Throughout testing and training sessions, the research team consistently instructed the subjects to maintain their same hand and foot placements.

\section{Statistical Analyses}

Descriptive statistics were calculated. A series of repeated measures ANOVAs (IBM SPSS version 22.0, IBM, Armonk, NY, USA) were used to compare muscle thickness at each site between the ST and HG groups across all three test times. Absolute change and percent change were reported for each group for each variable. Alpha was set a priori at 0.05 . 


\section{Results}

Fourteen subjects were encompassed in the analyses ( $\mathrm{SG} n=6 ; \mathrm{HG} n=8)$. At baseline, there were no statistically significant differences between groups for age, resistance training experiences, body mass, and height. Relative total average weekly

volume was $285.2 \pm 29.4 \mathrm{~kg} \cdot \mathrm{kg}^{-1}$ for SG and $236.0 \pm 61.9 \mathrm{~kg} \cdot \mathrm{kg}^{-1}$ for $\mathrm{HG}$ and were not statistically different $(p=0.24)$. Subjects reported no musculoskeletal injuries or impairments during or after completion of the experimental protocols.

\section{Muscle Thickness}

Positive values indicate an increase in muscle thickness and negative values indicate a decrease in muscle thickness. The percent change of muscle thickness can be seen in Table 3. 
Table 3

Muscle Thickness Measurements and Percent Change

\begin{tabular}{llllll}
\hline Measurement Site & Region & Pre & Mid & Post & \% Change \\
\hline SG Forearm Flexors & Proximal & $3.73(.21)$ & $3.79(.18)$ & $3.85(.20)$ & 3.2 \\
& Distal & $4.44(.27)$ & $4.34(.22)$ & $4.40(.27)$ & 1.1 \\
HG Forearm Flexors & Proximal & $3.36(.33)$ & $3.25(.26)$ & $3.21(.19)$ & -4.5 \\
& Distal & $4.00(.31)$ & $4.01(.21)$ & $4.03(.29)$ & 0.8 \\
SG Forearm Extensors & Proximal & $4.41(.63)$ & $4.55(.45)$ & $4.70(.51)$ & 6.6 \\
& Distal & $2.42(.42)$ & $2.63(.29)$ & $2.77(.33)$ & 14.5 \\
HG Forearm & Proximal & 4.21 & & & \\
Extensors & & $(1.05)$ & $4.19(.85)$ & $4.15(.90)$ & -1.4 \\
SG Rectus Femoris & Distal & $2.29(.35)$ & $2.27(.38)$ & $2.26(.44)$ & -1.3 \\
& Proximal & $2.90(.35)$ & $2.89(.33)$ & $2.92(.42)$ & 0.7 \\
HG Rectus Femoris & Distal & $2.60(.32)$ & $2.76(.31)$ & $2.71(.30)$ & 4.2 \\
& Proximal & $2.61(.45)$ & $2.67(.42)$ & $2.65(.41)$ & 1.5 \\
SG Vastus Lateralis & Distal & $2.73(.35)$ & $2.75(.33)$ & $2.73(.38)$ & 0.0 \\
& Proximal & & 3.39 & 3.37 & \\
& & $3.32(.97)$ & $(1.08)$ & $(1.17)$ & 1.5 \\
HG Vastus Lateralis & Distal & $2.69(.18)$ & $2.85(.21)$ & $2.89(.22)$ & 7.4 \\
& Proximal & 2.98 & 3.04 & 3.06 & \\
& & $(1.16)$ & $(1.03)$ & $(1.03)$ & 2.7 \\
& Distal & $2.57(.64)$ & $2.72(.65)$ & $2.78(.70)$ & 8.2
\end{tabular}

Note. Average muscle thickness measurements and standard deviations (shown in parentheses) in $\mathrm{cm}$ for both experimental groups over time. Percent change was calculated by subtracting the post-testing measurement by the pre-testing measurement, dividing the result by the same pre-testing measurement and multiplying the result by 100 for each measurement site.

Mean muscle thickness changes from baseline to post-testing for SG and $\mathrm{HG}$, respectively, are as follows: proximal FF: 3.2 vs. $-4.5 \%$; distal FF: 1.1 vs. .8\%; proximal FE: 6.6 vs. $-1.4 \%$; distal FE: 14.5 vs. $-1.3 \%$; proximal RF: .7 vs. $1.5 \%$; distal RF: 4.2 vs. 
$0.0 \%$; proximal VL: 1.5 vs. $2.7 \%$; distal VL: 7.4 vs. $8.2 \%$. Significant differences were observed in the proximal forearm flexors in favor of SG compared to HG at each point of testing $(p=.034, .001, .000)$. The distal FE measurements of both groups reached a significant difference at post-testing $(p=.036)$, but no significant differences were observed at other MT sites.

\section{Muscular Strength}

Similar strength values were achieved by the SG and HG during baseline testing of 1RM BS and 1RM BP. Over the experimental period, the SG increased their 1RM back squat from 126.5 to $146.2 \mathrm{~kg}$, the $\mathrm{HG}$ increased from 125.9 to $137.2 \mathrm{~kg}$. The $\mathrm{SG}$ increased their 1RM bench press from 101.9 to $113.2 \mathrm{~kg}$ and the HG increased from 89.6 to $92.9 \mathrm{~kg}$. After 9 weeks, the mean percent change of back squat for SG and HG, respectively, was $16.2 \%$ and $10.9 \%$. The percent change in bench press for SG was $12.7 \%$ and $4.6 \%$ for $\mathrm{HG}$.

\section{Discussion}

This research is among a small group that have compared two different volumeequated, total-body resistance training programs in well-trained men. Overall, only the PFF and DFE MT sites reached statistical significance between the experimental groups. As the SG experienced hypertrophy at the PFF and DFE MT sites, the hypertrophy group experienced a loss of muscle mass. This partially concurs with previous research demonstrating that skeletal muscle hypertrophy may be a non-uniform process, not only between the individual components of the muscle group, but also along the belly of each muscle (Narici, 1996). The strength group had an increase in muscle thickness over time at both PFF and DFE sites, but the hypertrophy group lost muscular thickness. This 
coincides with research showing that high-intensity resistance training may elicit significantly more hypertrophy than a moderate-intensity resistance training program (Mangine, 2015). Other research contradicts these claims and illustrate similar hypertrophy between high intensity and moderate intensity workloads when volume is equated (Schoenfeld, 2014). The lack of metabolic stress, mechanical tension and measurement error of the diagnostic ultrasound may have caused the decrease in MT observed in the HG.

Although there were no significant differences in any of the quadriceps muscle thickness comparisons, the 9-week length of the training program may not have been enough time to promote significant hypertrophy in the other MT sites. In a 20-week resistance training study by Chilibeck et al., they observed hypertrophy of the upper extremity muscles during the first 10 weeks and lower extremity and trunk muscle hypertrophy occurred during the last 10 weeks of the experimental procedure (1998). This may explain the lack of significant differences between the groups in the MT sites of the quadriceps.

In well-trained men, 9 weeks may not have been enough time to elicit statistically significant differences in hypertrophy between the experimental groups. Research has shown that well trained men may experience a desensitization to muscular hypertrophy when compared to their untrained counterparts, but more research is needed to confirm this phenomenon (Fisher, 2013). While not statistically significant, tendencies may have been seen between the groups among other forearm MT sites and should be reexamined under a longer protocol. 
Although a pattern may exist between the strength increases between the groups, no statistical significance was established. This contradicts the majority of literature stating greater strength improvements are achieved with higher relative intensity workloads, in comparison to a lesser relative intensity in trained and untrained individuals (Choi, 1998 and Schoenfeld, 2014). The lack of statistical significance may be due to the small sample size of the experiment.

The SG experienced greater hypertrophy with longer inter-set rest periods. Longer inter-set rest periods are associated with greater recovery and hypertrophy (Buresh, 2009 and Schoenfeld, 2015). Due to less metabolic stress, longer rest periods allow the subject to achieve more repetitions or work at a greater intensity. This results in higher mechanical stress on the muscle and ultimately greater hypertrophy. The results of this study support the notion that the added mechanical stress of longer inter-set rest periods, may outweigh the benefit of metabolic stress associated with hypertrophy training and shorter inter-set rest periods. Other research has argued that there are no difference or greater increases in hypertrophy with short rest periods in trained men (Ahtiainen, 2005).

The exercise organization and selection in a training protocol is crucial and significantly impacts how skeletal muscle adapts. One study that equated for volume, compared a total-body strength-style training program to a split-body hypertrophy-style training group using the same exercises. The study found that both groups hypertrophied similarly but the strength-style group significantly increased their strength over the hypertrophy-style group (Schoenfeld, 2014). The difference in exercise organization may explain why the hypertrophy group in the current study failed to experience the same 
hypertrophy that the strength group achieved. A training protocol that involves multiple sets of resistance exercises induces higher levels of growth hormone and testosterone in comparison with a single-set protocol (Antonio, 2000). The previous study using a split training protocol may have induced more hormonal changes and resulted in more hypertrophy than this current study utilizing total-body routines. In fact, Kraemer et al. (1990), investigated the effects of two different load and rest interval protocols on hormonal response in nine recreational resistance trainers. They discovered that serum testosterone increased regardless of the kind of training protocol. On the other-hand, serum growth hormone levels were highest using the protocol that consisted of high total work, 1 minute of rest, and 10RM load (Kraemer, 1990). Although significant hormonal changes are present, their effect on muscular hypertrophy has yet to be fully understood.

Also, each exercise activates the target muscles in a specific way which may lead to regional muscular hypertrophy. Research by Narici et al., as compared the site activation and hypertrophy of skeletal muscle and has determined a causative relationship exists between the level of activation during contractions and the degree of hypertrophy along a muscle belly in a non-uniform manner (1989). It would seem plausible that different exercises would stress different parts of a muscle and its muscle fibers. Perhaps this could alter gene expression with that particular myonucleus, resulting in the expression of particular myosin heavy-chain isoforms. Nonetheless, it is evident that skeletal muscle is heterogeneous between muscles, within a muscle, and within single muscle fibers (Antonio, 2000). The tracking of electromyographical activity in each MT site would benefit future research looking to replicate this study and the effects of regional activation on non-uniform hypertrophy. 
Some strengths of the current research should be noted here. First, the total-body exercise order allowed the observation of total-body routines and eliminated the effect of metabolic stress associated with split-body part routines. This may explain the negative effects on MT for subjects in the HG, indicating that total-body hypertrophy-style loading may not be an effective hypertrophy strategy. Second, no musculoskeletal injuries were reported during or after the 9-week training study. One similar research study had experienced a $10 \%$ subject mortality rate (Schoenfeld, 2014). The addition of a deloading week, in the middle of this study, may have helped to prevent injury. Third, this study may have discovered patterns that should be investigated using a longer resistance training protocol.

Several important limitations emerged throughout this study. First, the method used to calculate volume load (reps x load) did not calculate work performed correctly. The method does not take into consideration the distance travelled per load, per each exercise, or the forces applied during each of the movements. Secondly, the calculated volume load also was not similar to those used during a similar study (Schoenfeld, 2014). The load may not have been sufficient in causing enough of a hypertrophic response in the HG, an equal training load would have helped to examine this theory. Thirdly, the exercise selection for the lower-body included two exercises that were unfamiliar to most of the subjects in this study. Fourth, the 9 week training protocol was short and many MT measurements did not reach statistical significance. There were trends being seen and more measurements may have achieved significance if the study were longer. Fifth, although upper and lower-body areas were selected, the MT findings are not representative of all muscles or muscle groups of the subjects. There may have been 
hypertrophy occurring in areas such as the pectoralis major, latissimus dorsi, or biceps femoris. Lastly, the findings of this research study are specific to young, well-trained men and cannot be generalized to other populations. Further research should include a second HG that will follow a split-body routine, using the same 9 exercises. This would allow an examination on the effects of metabolic stress and mechanical tension on hypertrophy.

\section{Practical Application}

In conclusion, when a total-body routine is volume-equated, a high-intensity RT with long rest periods may have a greater effect on muscular hypertrophy than moderateintensity RT with short rest periods. Also, the significant differences found in the DFE and PFF indicate that these muscles hypertrophied in a non-uniform fashion. Nine weeks of the resistance training protocols may not have been long enough to identify significant changes at the other muscle sites. Further research is needed to understand the effects of different intensity, volume, rest intervals, and exercise selection and organization in welltrained men. 


\section{CHAPTER II}

\section{EXTENDED LITERATURE REVIEW}

\section{Introduction}

Skeletal muscular hypertrophy is of great interest to recreational gym goers, bodybuilders, older adults, persons suffering from immunodeficiency conditions and other populations seeking an increase in skeletal muscle. Current research has established that properly regimented resistance training can promote muscular hypertrophy (Schoenfeld, 2010). Skeletal muscle is an ever-changing tissue and adapts to the demands and strains of resistance training. After several weeks of progressive resistance exercise, increases in muscular strength and hypertrophy are typically observed (American College of Sports Medicine, 2009).

The act of hypertrophy is mediated by mechanotransduction, whereby sarcolemmal-bound mechanosensors, convert mechanical forces into chemical signals that regulate the activation of anabolic and catabolic pathways. When sufficient mechanical overload is induced, anabolic processes prevail over catabolic processes to promote a net increase in muscle protein synthesis and corresponding enlargement of fibers (Schoenfeld, 2015). Pathways that have been identified as particularly important to muscle anabolism include mammalian target of rapamycin (mTOR), mitogen-activated protein kinase (MAPK), and various calcium-dependent pathways, amongst others. Many of these anabolic signaling pathways are involved in exercise induced gains in 
muscle mass with certain pathways functioning in a permissive role while others directly mediate cellular processes that influence messenger RNA (mRNA) translation and thus hypertrophy (muscle cell growth) (Schoenfeld, 2013).

Hypertrophy is non-universal throughout the body and different forms occur due to different manipulations of exercise variables (Antonio, 2000). In fact, the types of contraction utilized may result in different types of muscular hypertrophy. Eccentric contraction loading has been shown to be associated with an increase in the series of sarcomeres in a muscle and concentric exercises are associated with a shortening of the sarcomere length. Eccentric contractions influence this by lengthening the muscle belly and forcing the muscle to adapt to the new functional length of the muscle. The increase of sarcomeres and myofibrils added in parallel is how powerlifters and traditional resistance trained individuals hypertrophy (Schoenfeld, 2010). Another form of hypertrophy, called sarcoplasmic hypertrophy, is an increase in various non-contractile elements and fluid. This hypertrophy is considered nonfunctional due to the lack of strength increases and is common amongst bodybuilders (Schoenfeld, 2010).

Overall, muscle hypertrophy occurs when protein synthesis exceeds protein break-down (Schoenfeld, 2010). To initialize these hypertrophic adaptations, exercise programming must properly manipulate volume, intensity, rest period, exercise selection and exercise organization. Programming of said variables results in different amounts of metabolic damage, mechanical stress, recovery, and regional activation throughout the exercises on the involved muscles (Schoenfeld, 2014). The importance of understanding how to manipulate these influences is critical in mediating the desired type, amount and region of muscular hypertrophy. 


\section{Volume}

Volume is a key mediator in skeletal muscle hypertrophy. Exercise volume is the total product of the repetitions, sets, and the workload for a given exercise or exercise program. A training protocol that involves multiple sets of resistance exercises induces higher levels of growth hormone and testosterone in comparison with a single-set protocol (Antonio, 2000). This adds support to the notion that more volume may equate to more metabolic stress. Metabolic stress pursuant to exercise manifests as a result of the accumulation of metabolites, particularly lactate, $\mathrm{Pi}$ and $\mathrm{H}+$, calcium, and various electrolytes. Acute muscle hypoxia associated with resistance training may serve to further heighten metabolic buildup and, hence, stimulate hypertrophic adaptations (Schoenfeld, 2015).

Support for the potential hypertrophic role of exercise induced metabolic stress can be noted empirically by examining the moderate-intensity training regimens adopted by a majority of bodybuilders, which are intended to heighten metabolic buildup at the expense of higher training intensities (Schoenfeld, 2013). Typical hypertrophy-oriented bodybuilding routines involve the performance of multiple sets of 6-12 repetitions per set with relatively short inter-set rest intervals. These routines have been found to induce significantly more metabolic stress than higher intensity regimens typically employed by powerlifters. Powerlifters often train with heavy loads for 5, or less, repetitions and taking at least 3 minutes between sets using several structural exercises during specific strength training phases (Schoenfeld, 2014). Several studies have reported greater increases in muscle growth from moderate-intensity bodybuilding-type training protocols as compared with high-intensity powerlifting-style routines, although these findings are 
not consistent across all trials when equating for volume load (Schoenfeld, 2013). This places the importance on research equating volume between different resistance training programs.

In an exercise program, volume can be organized in either a split-body or totalbody routine. The goal of a split-body routine is to create as much metabolic and mechanical stress on a group of muscles in a single workout. This is thought to maximize hypertrophic adaptations and allow recovery for the muscle throughout the rest of the week. This results in less exercises performed per session and less time in the gym (Schoenfeld, 2014). In contrast to a split-body routine, the goal of a total-body routine is to target all or most of the muscles in the body during a single workout session. This limits the metabolic and mechanical damage done to a muscle and allows for more frequent training sessions of a muscle group during the week (Schoenfeld, 2014).

The training status of an individual greatly influences the amount of volume that they can utilize to maximize hypertrophy (American College of Sports Medicine, 2009). Over time, an improvement in neuromuscular and hypertrophic adaptations, allows for an individual to increase their resistance training volume. This increase is necessary to recruit and stimulate the same amount or more muscle fibers (American College of Sports Medicine, 2009). As resistance training experience increases, the ability to obtain muscle mass becomes gradually more difficult (Schoenfeld, 2010).

A recent study by Schoenfeld et al. (2014), examined the effects of two different volume-equated different resistance training programs on well trained men. Over nine weeks, one group followed a strength-style of training and the other followed a hypertrophy-style of training. While each group completed the same 9 exercises, the 
strength group followed a total-body protocol and the hypertrophy group followed a splitbody protocol. The results indicated that both groups achieved similar results in hypertrophy, but the strength group achieved a higher strength total. The hypertrophy results are in accordance with previous studies in untrained subjects that controlled for volume, but are in disagreement with those that did not control for volume in untrained subjects (Schoenfeld, 2014).

\section{Intensity}

Intensity is the workload of an exercise that is relative to the one-repetition maximum of the individual completing the exercise. Intensity is customarily expressed as a percentage of 1RM and equates to the number of repetitions that can be performed with a given weight. Repetitions can be classified into 3 basic ranges: low (1-5), moderate (6-12), and high (15+). Each of these repetition ranges will involve the use of different energy systems and tax the neuromuscular system in different ways, impacting the extent of the hypertrophic response (Schoenfeld, 2010). Evidence suggests that both high and low intensities stimulate hypertrophy and maximize the enrollment of muscle fibers when training to momentary muscle failure (Antonio, 2000). Although high rep training can bring about significant metabolic stress, especially when combined with occlusion training, the load fails to recruit and fatigue the maximum amount of motor units. While training with low to moderate rep ranges stimulate the hypertrophic response, there is conflicting data on which rep range is best suited to induce optimal muscular hypertrophy (Schoenfeld, 2010).

The benefit of moderate repetitions has been attributed to metabolic stress due to the heavy reliance upon anaerobic glycolysis. This results in a significant buildup of 
blood lactate, intramuscular lactate, glucose, glucose- 6-phosphate and other metabolites; while simultaneously depleting ATP, creatine phosphate and glycogen (Schoenfeld, 2010). The accumulation of these metabolites have been shown to impact anabolic processes and stresses the importance of metabolic factors. Resultant to metabolic buildup, moderate repetition range training has been shown to maximize the acute anabolic hormonal response of exercise. Both testosterone and GH are acutely elevated to a greater degree from routines employing moderate rep sets as compared to those using lower repetitions (Kraemer, 1990 and Schoenfeld 2010), thereby increasing the potential for downstream cellular interactions that facilitate remodeling of muscle tissue.

Training in a moderate repetition range also maximizes acute cellular hydration and a buildup of fluid into interstitial spaces. This results in cell swelling and the commonly achieved "pump" while weight training (Schoenfeld, 2010). Although the hypertrophic impacts of acute exercise-induced cell swelling are unknown, it seems possible that the extra cell hydration may lead to a resistance training benefit. Another benefit associated with moderate rep protocols, in comparison to a low rep scheme, is the extra time under tension. This could enhance the potential for micro trauma and fatigueability across the entire spectrum of muscle fibers (Schoenfeld, 2010). While many human muscles contain approximately 50\% fast and 50\% slow fibers it is evident that there are certain muscles that may be primarily fast or slow. The added benefit of extra time under tension would best apply to slow-twitch fibers that have a greater endurance capacity, but are less responsive to growth in comparison to fast-twitch fibers (Antonio, 2000). Some researchers have theorized that muscles containing a greater percentage of slow-twitch fibers might have the greatest hypertrophic response to a higher repetition 
range, whereas fast-twitch muscles would respond best to lower repetitions. Although this concept is intriguing, a fiber-type prescription with respect to repetition range has not been examined. Also, given the variability of fiber-type composition between individuals, it would be near impossible to determine fiber-type ratios without muscle biopsy (Schoenfeld, 2010).

As intensity increases, the amount of time needed to rest between sets also increases. A study by Schoenfeld et al., examined the effects of two different rest periods on a hypertrophy-style total-body resistance training. The 21 well-trained male subjects completed the same seven exercises on non-consecutive days, for 8 weeks. One group were allotted 1 minute rest periods and the other was allowed three minute rest periods. After the experiment was completed, the 3 minute rest periods experienced a greater increase in hypertrophy and strength. This follows suit with research examining optimal strength results, but is in contrast to most optimal hypertrophy protocols. While there was no statistical significant difference in volume between the groups, the 3 minute group achieved a slightly higher work volume. This higher work volume may account for the increases in strength and hypertrophy (2015).

\section{Non-Uniform Hypertrophy}

Current research has shown muscular hypertrophy results in a non-uniform fashion. Non-uniform muscular hypertrophy is the regional-specific hypertrophic response of a muscle or muscle groups. In fact, the idea that a muscle would respond in a uniform fashion would seem implausible in light of the fact that there are distinct physiological and anatomical differences within a single muscle (Antonio, 2000). Skeletal muscle is a heterogeneous tissue that is composed of 2 basic fiber types: fast- 
twitch and slow-twitch. These fiber types have been categorized more specifically on the basis of $\mathrm{pH}$ lability of myofibrillar (myosin) ATPase reaction, glycolytic/oxidative capacities, or myosin heavy chain (MHC) isoform content. Each classification explains the individual responsibilities and reactions of the fibers to mechanical stress. If enough consistent mechanical stress is placed upon a muscle fiber, the dynamic ability of muscle fibers allows it to adapt and better suit the demand. This adaptation and specific growth of muscle fibers may contribute to regional muscle hypertrophy (Antonio, 2000). Although specific muscle fiber training has yet to be understood and may pose significant challenges. Some individuals find biopsies too invasive and determining fiber typing without the use of biopsies is near impossible.

Furthermore, current research has extrapolated the relationship between regional muscle activation and hypertrophy. Research examining both EMG activity and regional hypertrophy have shown a linear relationship. Single vs compound exercises, grip variation, structural differences, compartmentalization and muscle fiber typing may play a significant role in non-uniform hypertrophy (Antonio, 2000). Wakahara et al. examined the effects of muscle activation on non-uniform muscle hypertrophy. The subjects of the study participated in a 12 week resistance training program for the left upper limb. The highest achieved activation occurred in the middle of the triceps, which also fostered the most hypertrophy along the muscle bellies (2013). This strongly suggests that the regional activation of muscle is at least partially responsible for the nonuniform hypertrophy phenomenon. 


\section{Gaps in the Literature}

RT has established that exercise volume is an important component to skeletal muscle hypertrophy. Controlling for intensity and volume has allowed us to examine resistance training variables more closely, but there has yet to be definitive evidence on the optimal training program for inducing muscular hypertrophy. Even less understood, is the effect on well-trained or even professional athletes who benefit from resistance training. Research must further explore resistance training variables by completing longer and more detailed experiments. 


\section{REFERENCES}

Ahtiainen JP, Pakarinen A, Alen M, Kraemer WJ, and Hakkinen K. Short vs. long rest period between the sets in hypertrophic resistance training: influence on muscle strength, size, and hormonal adaptations in trained men. J Strength Cond Res 19: 572-582, 2005.

American College of Sports Medicine. American College of Sports Medicine position stand. Progression models in resistance training for healthy adults. Med Sci Sports Exerc 41(3): 687-708, 2009.

Antonio, J. Nonuniform Response of Skeletal Muscle to Heavy Resistance Training: Can Bodybuilders Induce Regional Muscle Hypertrophy? J Strength Cond Res 14: 102-113, 2000.

Baechle, TR and Earle, RW. Essentials of Strength and Conditioning. In: Anonymous. Champaign, IL: Human Kinetics, 2008.

Buresh R, Berg K, and French J. The effect of resistive exercise rest interval on hormonal response, strength, and hypertrophy. J Strength Cond Res 23: 62-71, 2009.

Chilibeck P.D., Calder A.W., Sale D.G., and Webber C.E, A comparison of strength and muscle mass increases during resistance training in young women. Eur. J. Appl. Physiol. 77:170-175, 1998.

Choi J, Takahashi H, Itai Y, and Takamatsu K. The difference between effects of "powerup type" and "bulk-up type" strength training exercises: With special reference to muscle cross-sectional area. Jpn J Phys Fitness Sports Med 47: 119-129, 1998.

Fisher J, Steele J, Smith D. Evidence-based resistance training recommendations for muscular hypertrophy. Med Sport 17 (4): 217-235, 2013.

Kraemer, W.J., Marchitelli L, Gordon S.E., Harman E., Dziados J.E., Mello R, Frykman P., McCurry D, and Fleck S.J. Hormonal and growth factor responses to heavy resistance exercise protocols. J. Appl. Physiol. 69:1442-1450, 1990. 
Mangine GT, Hoffman JR, Gonzalez AM, Townsend JR, Wells AJ, Jajtner AR, Beyer KS, Boone CH, Miramonti AA, Wang R, LaMonica MB, Fukuda DH, Ratamess NA, and Stout JR. The effect of training volume and intensity on improvements in muscular strength and size in resistance-trained men. Physiol Rep 3(8): e12472, 2015.

Narici, M.V., G.S. Roi, L. Landoni, A.E. Minetti, and P. Cerretelli. Change in force, cross-sectional area and neural activation during strength training and detraining of the human quadriceps. Eur. J. Appl. Physiol. 59:310-319. 1989.

Narici M.V., Hoppeler H, Kayser B, Landoni L, Claassen H., Gavardi C, Conti M, and Cerretelli P. Human quadriceps cross-sectional area, torque, and neural activation during 6 months of strength training. Acta Physiol. Scand. 157:175-186. 1996.

Schoenfeld BJ. The mechanisms of muscle hypertrophy and their application to resistance training. J Strength Cond Res 24(10): 2857-2872, 2010.

Schoenfeld BJ. Is there a minimum intensity threshold for resistance training-induced hypertrophic adaptations? Sports Med 43: 1279-1288, 2013.

Schoenfeld, BJ, Pope, ZK, Benik, FM, Hester, GM, Sellers, J, Nooner, JL, Schnaiter, JA, Bond-Williams, KE, Carter, AS, Ross, CL, Just, BL, Henselmans, M, and Krieger JW. Longer inter-set rest periods enhance muscle strength and hypertrophy in resistance-trained men. J Strength Cond Res. Advance online publication. doi: 10.1519/JSC.0000000000001272. 2015.

Schoenfeld BJ, Ratamess NA, Peterson MD, Contreras B, Sonmez GT, and Alvar BA. Effects of different volume-equated resistance training loading strategies on muscular adaptations in well-trained men. J Strength Cond Res 28(10): 2909-18, 2014.

Wakahara T, Fukutani A, Kawakai Y, Yani T. Nonuniform MuscleHypertrophy: Its Relation to Muscle Activation in Training Session. Med Sci Sports Exerc 45: 2158-2165, 2013. 